\title{
Damage Process Simulation of Damping Coating of Thin Plate
}

\author{
Jingyu Zhai ${ }^{\mathrm{a}}$, Hui Li ${ }^{\mathrm{b}}$ and Qingkai Han * ${ }^{\mathrm{c}}$ \\ ${ }^{1}$ School of Mechanical Engineering and Automation, Northeastern University, \\ Shenyang, PR China, 110819 \\ azhaijingyu_neu@163.com, ${ }^{\text {b2 } 24705928 @ q q . c o m, ~ * ~ c ~ q h a n @ m a i l . n e u . e d u . c n ~(C o r r e s p o n d i n g ~ a u t h o r) ~}$
}

Keywords: Thin plate, damping coating, damage process simulation, stress intensity factor

\begin{abstract}
In this paper, the element birth and death technique is used to simulate the damping stripping process of damping coating, and the interface of substrate and damping coating is simulated by contact elements or interface elements. The stress intensity factors and crack length are also calculated based on finite element method under different thicknesses and elastic modulus The simulation could provide reference for the design and optimize of damping coating.
\end{abstract}

\section{Introduction}

Damping coating on the surfaces of composite structure, which is made from different materials, can improve their dynamic performance, thus reduce vibration stress and lighten fatigue failure of the thin-walled structural member such as blade. But, the failure mechanisms and damage processes of damping coating have not been clear at present. The reliability of damping coating was related to the security of the whole components, and the coating characteristics were critical to the widespread application of coatings.

The cracks are easily initiated from the interface of the substrate and damping coating. The crack propagation usually lead to the failure of coating [1-2]. It is essential to simulate the damage process of damping coating and study its effective factors.

In this paper, the element birth and death technique is uses to simulate the damping stripping process of damping coating, and the stress intensity factors and crack length are calculated by using finite element method under different thicknesses and elastic modulus.

\section{FEM simulation of fatigue crack growth}

In ANSYS, the interface element and contact element are used to simulate the crack zone and the approach for crack propagation by killing or 'death' elements is introduced. The process of deactivating the element properties is described in details in [3]. In this method, the formation and the propagation of the crack are modeled by using the interface element.

The non-linear truss elements, which have different material properties is used to connect the boundary nodes ahead of the initial crack tip, as shown in Fig. 1. These elements have the same cross-sectional area and have capabilities to take both tension and compression loads. The stiffness of each truss element in terms of modulus of elasticity has different values from an extremely large value (other end from the crack tip) to a value near the yield stress (near crack tip). The elemental axial stress is calculated as the load is applied on the structure. When any element's stress exceeds the critical bonding stress that element is killed (deactivated material property) by using EKILL command. At the same time, the element material properties from the crack tip to the other end are moved (changed) successively.

In this paper, the interface is simulated by contact elements. The simulation is carried out through solving the contact problems. Modeling with contact elements involves the same steps as any other contact analysis. 

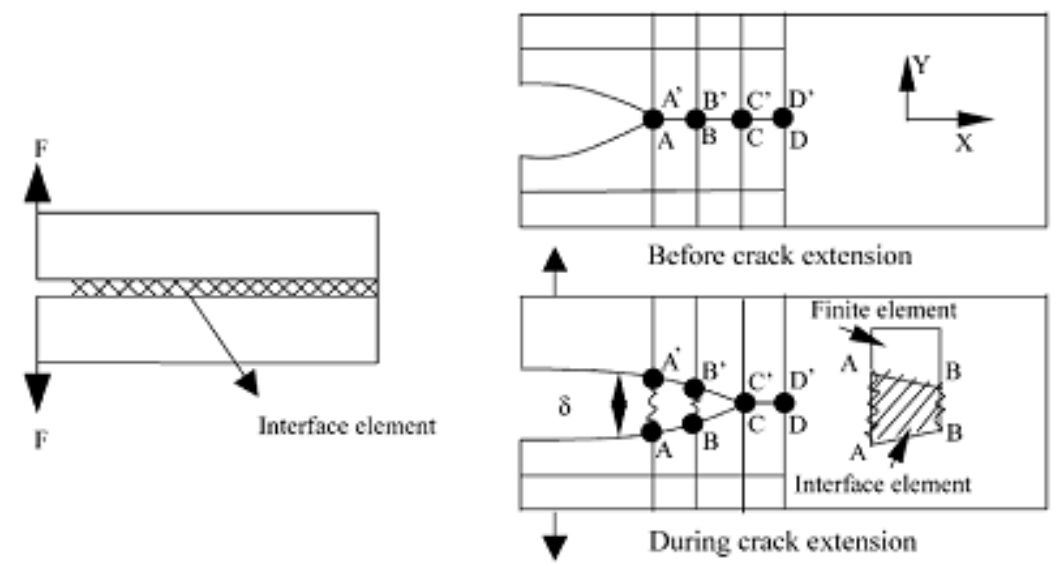

\section{Case studies}

Fig.1. Interface element between crack faces

To calculate the Fracture parameters and simulate the damage process, the thin plate with damping coating is simplified as a plane-stress problem. A 2D model of a thin plate with damping coating is created, as shown in Fig.2. The model is meshed with plane 182 element, and contact elements are applied in the connect faces, which are also the crack faces, as shown in Fig.3. The left side of the model is fixed in all degrees of freedom, and the translation degrees of freedom of bottom side are fixed in $\mathrm{x}$, y direction. A slope load is applied at the surface of damping coating, as shown in Fig.4.

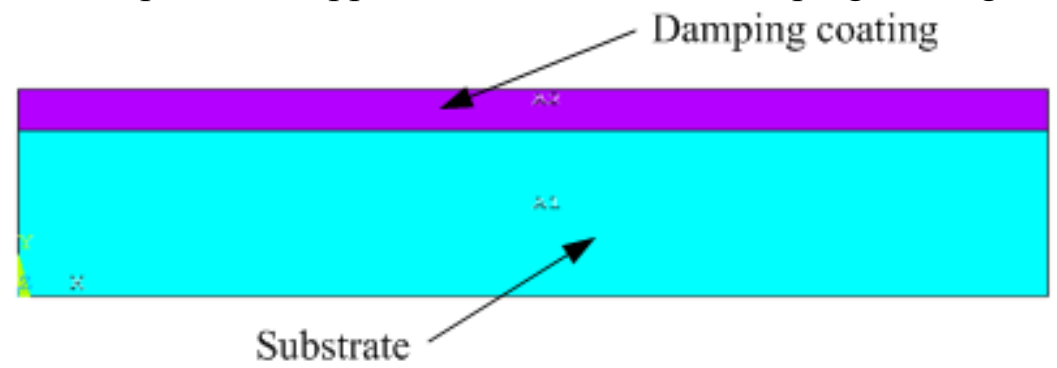

Fig. 2. The 2D model of a thin plate with damping coating

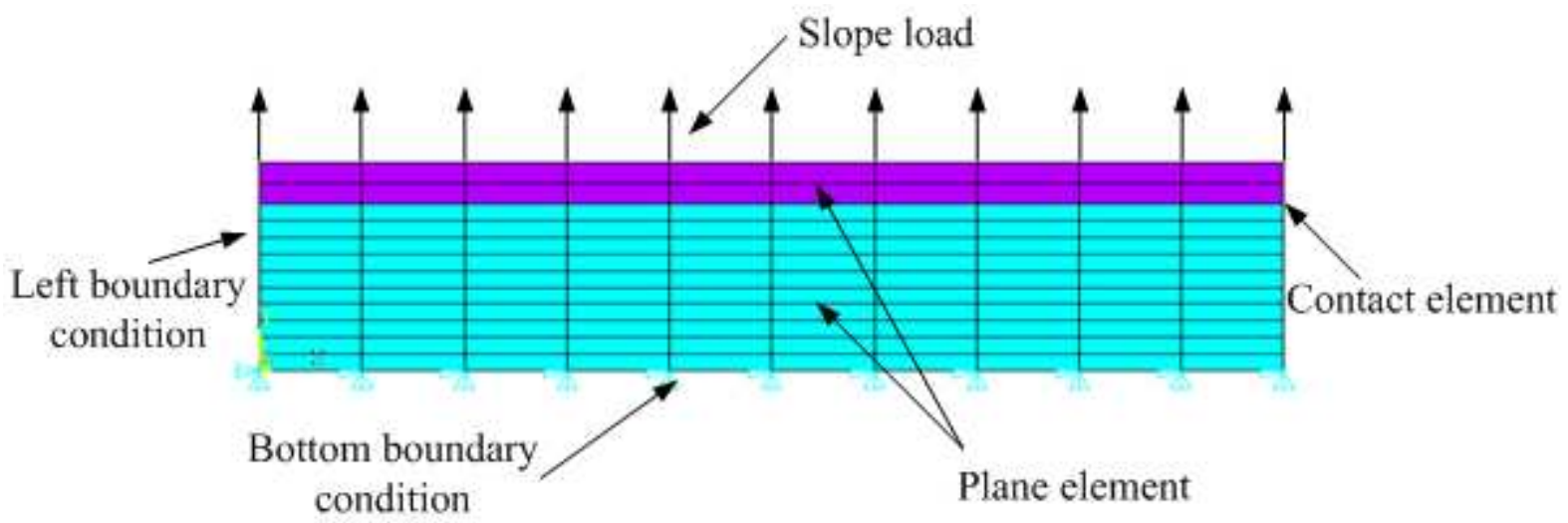

Fig. 3. The finite model of a thin plate with damping coating

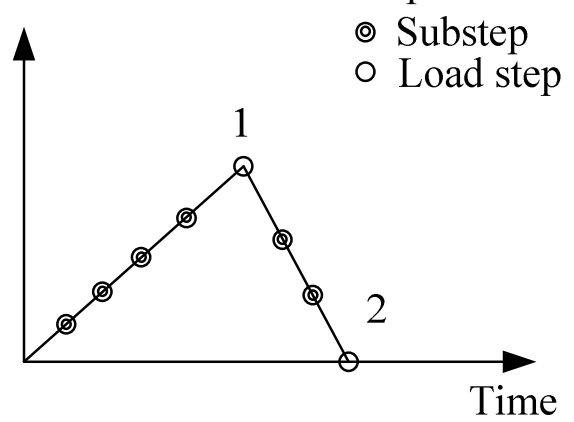

Fig. 4. The ramped load 
The material parameters of the thin plate and damping coating as shown in Table 1 are applied to the model. The NiCrAlY is selected as the material of damping coating.

Table1 Material parameters of the thin plate with damping coating

\begin{tabular}{|c|c|c|}
\hline Material & Modulus of elasticity $(\mathrm{GPa})$ & Poisson's ratio \\
\hline Metal substrate & 212 & 0.288 \\
\hline Damping coating (NiCrAlY) & 96.3 & 0.31 \\
\hline
\end{tabular}

\section{Results and discussions}

The stress intensity factor indicates the strength of the singular stress field at crack tip, it is the basis of studying the law of crack propagating and the strength of the component with crack. The value of it is related to the crack length, the physical dimension of structure and the external force, so the thicknesses of damping coating will effect the fracture properties of composite structure.

The ratios of thicknesses of substrate and damping coating are set as $1,2,5,10$. The stress intensity factors are calculated with different thicknesses ratios, as shown in Fig.5. The stress intensity factors I and II changes with the thicknesses ratio of substrate and damping coating. But the effective law is different. The stress intensity factor I increases with the thicknesses ratio, but the the stress intensity factor II decreases firstly, and then increases.

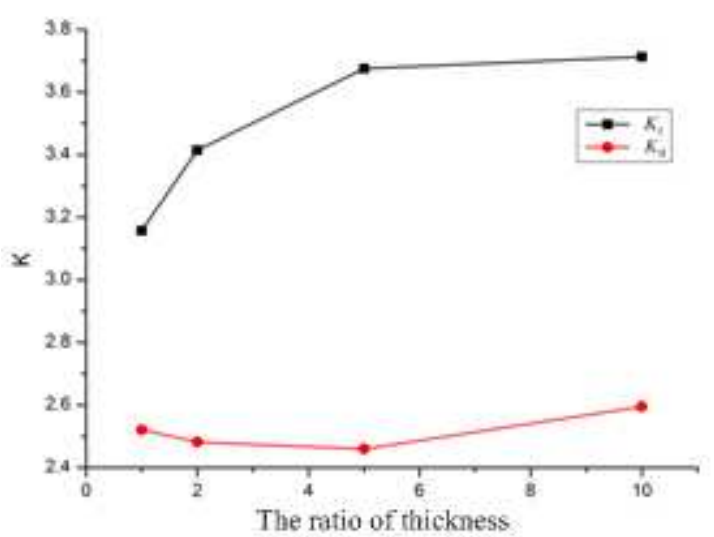

Fig. 5. Thickness ratio vs stress intensity factors

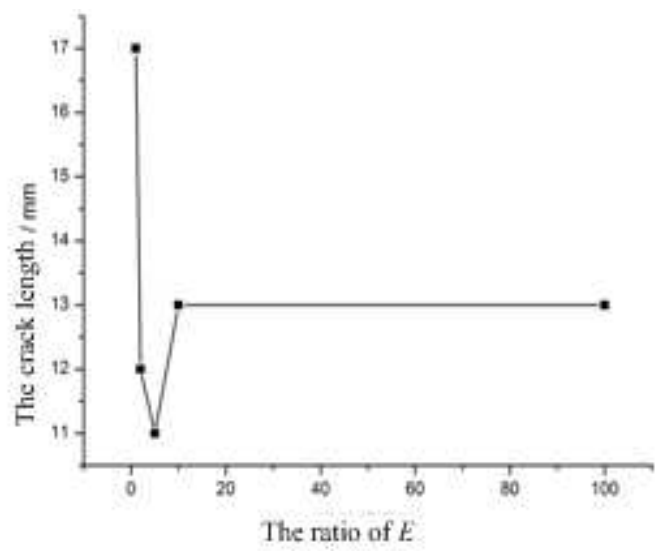

Fig. 6. Elasticity modulus vs crack length

To investigate the effects of different damping coating materials to the fracture properties of composite structure, the crack lengths with different elastic modulus are calculated. The material of substrate is shown in Table 1. The elastic modulus ratios of materials of substrate and damping coating are set as 1, 5, 10, 100. The relationship of the ratio and the crack length is shown in Fig.6. When the ratio is more than 10, the crack length stays the same, although the initiation and propagation of crack are related to many factors.

In this paper, the damage process of thin plane with damping coating is simulated based on the element birth and death technique. As in the practical application, the structures of substrate and coating depend on the preparation methods of damping coating. The model is simplified in this paper, and the aim of simulation is to obtain the fracture form, as shown in Fig.7. 


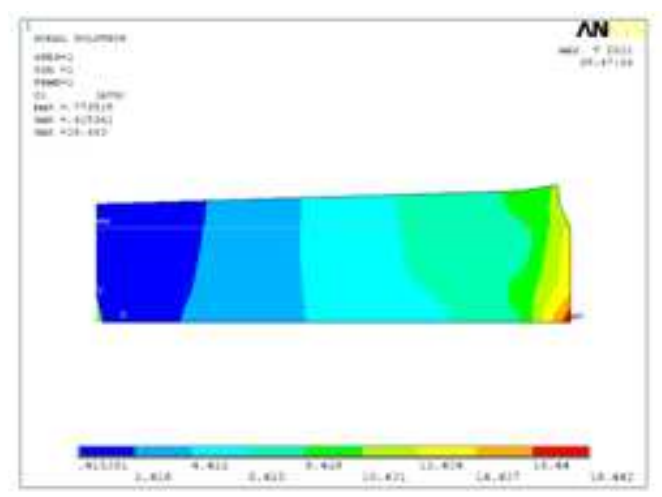

(a)

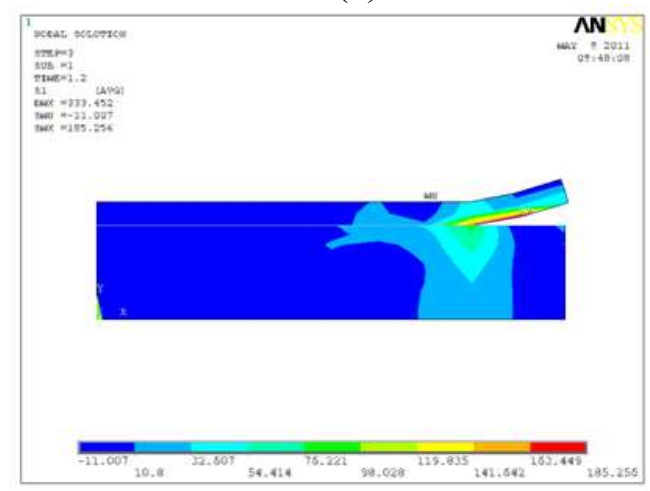

(c)

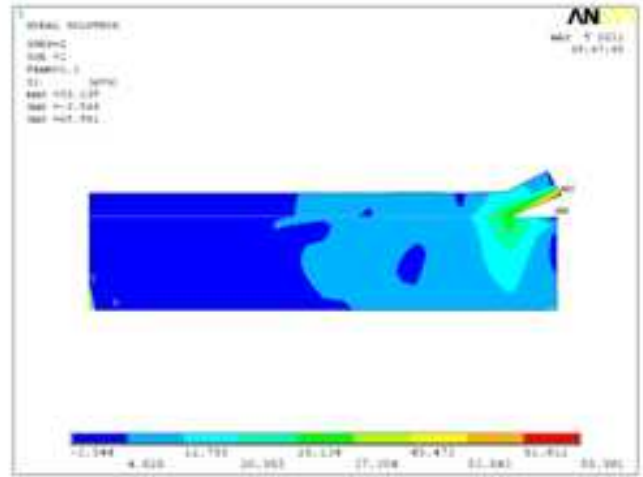

(b)

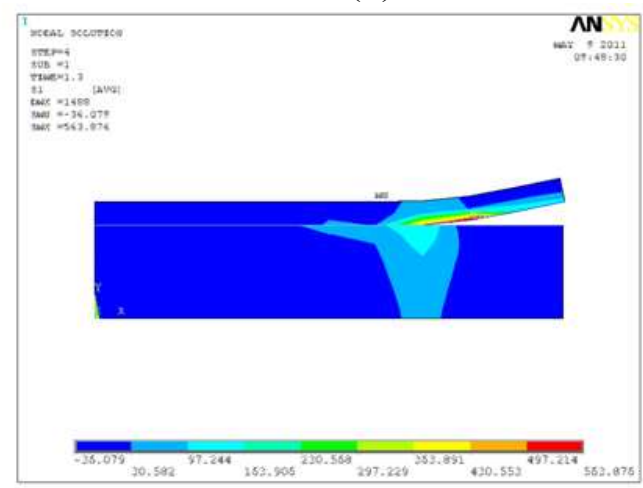

(d)

Fig. 7. Damage process simulation of the thin plane with damping coating

\section{Conclusions}

The proposed simulation of damage process of thin plane with damping coating is carried out based on the element birth and death technique. The stress intensity factors and the crack length are calculated to study the effects of the thickness and the materials. The simulation could provide reference for the design and optimize of damping coating.

\section{Acknowledgements}

This work was financially supported by the Fundamental Research Funds for the Central Universities (N090603007).

\section{References}

[1] L. Liu, X.G. Yang. 1]he Analysis of the Fracture Parameters of the Functional Thermal Barrier Coating, Aeroengine. 4 (2003) 22-24.

[2] W.L. Wei, X.G. Yang, H.Y. Qi. Numerical simulation of damage and fracture on typical interfaces of plasma sprayed thermal barrier coatings, Journal of Beijing University of Aeronautics and Astronautics. 10 (2007) 1141-1145.

[3] M.S. Alam, M.A. Wahab. Modeling the fatigue crack growth and propagation life of a joint of two elastic materials using interface elements, Pressure Vessels and Piping. 82 (2004) 105-113.

[4] X.D. Li, Y. Zhang, F.W. Zhang, D.H. Zhang, Z.P. Li. Finite element research on the effect of interfacial bond strength on fracturing process of ceramic matrix composites, Acta Materiae Compositae Sinica. 5 (2009) 132-136.

[5] V.V. Zozulya, C.I. Gouzalez. Dynamic fracture mechanics with contact interaction at the crack edges, Engineering Analysis with boundary elements. 24 (2004) 643-659. 